\title{
(6) OPEN ACCESS \\ Therapeutic hypothermia initiated within 6 hours of birth is associated with reduced brain injury on MR biomarkers in mild hypoxic-ischaemic encephalopathy: a non-randomised cohort study
}

\author{
Paolo Montaldo, ${ }^{1}$ Peter J Lally, ${ }^{1}$ Vânia Oliveira, ${ }^{1}$ Ravi Swamy, ${ }_{1}^{1}$ Josephine Mendoza, ${ }^{1}$ \\ Gaurav Atreja, ${ }^{2}$ Ujwal Kariholu, ${ }^{2}$ Vijayakumar Shivamurthappa, ${ }^{2}$ Natasha Liow, ${ }^{2}$ \\ Justinas Teiserskas, ${ }^{2}$ Russell Pryce, ${ }_{1}^{3}$ Aung Soe, ${ }^{3}$ Seetha Shankaran, ${ }_{1}^{4}$ Sudhin Thayyil ${ }^{1}$
}

- Additional material is

published online only. To view please visit the journal online (http://dx.doi.org/10.1136/ archdischild-2018-316040).

${ }^{1}$ Centre for Perinatal Neuroscience, Imperial College London, London, UK ${ }^{2}$ Neonatal Unit, Imperial Healthcare NHS Trust, London, UK

${ }^{3}$ Oliver Fisher Neonatal Unit, Medway NHS Hospital Foundation Trust, Gillingham UK

${ }^{4}$ Perinatal-Neonatal Medicine, Wayne State University, Detroit, Michigan, USA

Correspondence to Dr Sudhin Thayyil, Department of Paediatrics, Centre for Perinatal Neuroscience, Imperial College London, London W12 OHS, UK s.thayyil@imperial.ac.uk

Received 13 August 2018 Revised 22 October 2018 Accepted 23 October 2018 Published Online First 13 November 2018

\section{ABSTRACT}

Objective To examine the effect of therapeutic hypothermia on MR biomarkers and neurodevelopmental outcomes in babies with mild hypoxic-ischaemic encephalopathy (HIE).

Design Non-randomised cohort study.

Setting Eight tertiary neonatal units in the UK and the USA.

Patients 47 babies with mild HIE on NICHD

neurological examination performed within 6 hours after birth.

Interventions Whole-body cooling for 72 hours $(n=32)$ or usual care $(n=15$; of these 5 were cooled for $<12$ hours).

Main outcome measures MRI and MR spectroscopy (MRS) within 2 weeks after birth, and a neurodevelopmental outcome assessment at 2 years. Results The baseline characteristics in both groups were similar except for lower 10 min Apgar scores $(p=0.02)$ in the cooled babies. Despite this, the mean (SD) thalamic NAA/Cr (1.4 (0.1) vs $1.6(0.2) ; p<0.001)$ and NAA/Cho (0.67 (0.08) vs 0.89 (0.11); $p<0.001)$ ratios from MRS were significantly higher in the cooled group. Cooled babies had lower white matter injury scores than non-cooled babies $(p=0.02)$. Four $(27 \%)$ non-cooled babies with mild HIE developed seizures after 6 hours of age, while none of the cooled babies developed seizures $(p=0.008)$. Neurodevelopmental outcomes at 2 years were available in $40(85 \%)$ of the babies. Adverse outcomes were seen in $2(14.3 \%)$ noncooled babies, and none of the cooled babies $(p=0.09)$. Conclusions Therapeutic hypothermia may have a neuroprotective effect in babies with mild HIE, as demonstrated by improved MRS biomarkers and reduced white matter injury on MRI. This may warrant further evaluation in adequately powered randomised controlled trials.

\section{INTRODUCTION}

(c) Author(s) (or their employer(s)) 2019. Re-use permitted under CC BY. Published by BMJ.

To cite: Montaldo P, Lally PJ, Oliveira V, et al. Arch Dis Child Fetal Neonatal Ed 2019:104:F515-F520.
Several major cooling trials in the past decade provide conclusive evidence for the safety and efficacy of cooling therapy in improving survival without disability after moderate or severe hypoxic-ischaemic encephalopathy (HIE) in high-income countries. ${ }^{1}$ These trials excluded babies with mild HIE, and hence the optimal neuroprotective

\section{What is already known on this topic?}

Therapeutic hypothermia for 72 hours reduces brain injury on neonatal MRI and improves later neurodevelopmental outcomes after moderate or severe hypoxic-ischaemic encephalopathy (HIE).

- Despite a lack of evidence, many cooling centres in the UK and in other high-income countries routinely cool babies with mild HIE.

\section{What this study adds?}

Whole-body cooling initiated within 6 hours of birth and continued for 72 hours reduces cerebral metabolite perturbations and white matter brain injury seen on MRI.

- A small proportion of the non-cooled babies with mild HIE may develop seizures after 6 hours of age and progress to moderate HIE.

strategies for these babies remain unclear. Although adverse neurodevelopmental outcomes at 2 years or more are seen in up to $25 \%$ of infants with mild HIE, pooled data from clinical trials involving 117 babies fail to show any benefits of therapeutic hypothermia in mild HIE. ${ }^{2}$ Despite this uncertainty, cooling therapy has crept into the routine management of babies with mild HIE. ${ }^{3}$

MRI and MR spectroscopy (MRS) biomarkers can accurately quantify brain injury and assess the treatment effects of neuroprotective therapies in HIE with greater power than clinical outcome measures, and hence require reduced sample sizes. ${ }^{4}$ Unlike moderate or severe HIE, babies with mild HIE often have white matter injury, and deep brain nuclei injury is often not visible on conventional MRI. ${ }^{5}$ However, the thalamus is one of the most metabolically active areas in the fetal brain, and hence some degree of apoptosis is invariably seen in the thalami in preclinical models even with milder ischaemic insults. ${ }^{6}$ Thalamic proton MRS is exquisitely sensitive to these changes.

The aim of this observational study was to examine the effect of whole-body hypothermia on 
cerebral MR biomarkers and neurological outcomes at 2 years after mild HIE.

\section{METHODS}

We identified babies with mild HIE who were recruited as part of the large international, prospective multicentre Magnetic Resonance Biomarkers in Neonatal Encephalopathy (MARBLE) study between January 2013 and June 2016. ${ }^{7}$

Consecutive full-term ( $\geq 36$ weeks) infants admitted to the neonatal intensive care unit with mild HIE following a perinatal hypoxic event were included in this substudy of MARBLE. We accepted any of the following as evidence of perinatal asphyxia: metabolic acidosis on cord/baby blood gas within 1 hour of birth; an acute intrapartum event (eg, cord prolapse, abruption, antepartum bleed); a 10 min Apgar score $\leq 5$; or assisted ventilation initiated at birth and continued $\geq 10 \mathrm{~min}$.

Babies who fulfilled these criteria underwent a standardised neurological examination within 6 hours of birth using the NICHD Neonatal Research Network trial of hypothermia, by a certified examiner, with extended criteria to include mild HIE. ${ }^{8}$ We defined mild HIE as infants with $\geq 2$ abnormal categories but with no evidence of moderate or severe HIE (defined as moderate and/or severe abnormality in $\geq 3$ categories).

All babies had amplitude integrated electroencephalography (aEEG) for at least the first 6 hours after birth. We classified the aEEG background activity as normal (the upper margin of band of aEEG activity $>10 \mu \mathrm{V}$ and the lower margin $>5 \mu \mathrm{V}$ ), moderately abnormal amplitude (the upper margin of band of aEEG activity $>10 \mu \mathrm{V}$ and the lower margin $<5 \mu \mathrm{V}$ ), or severely abnormal amplitude (the upper margin of the band of aEEG activity $<10 \mu \mathrm{V}$ and lower margin $<5 \mu \mathrm{V}$ ). Infants were divided into two groups: those who received 72 hours of therapeutic hypothermia and those who did not receive any hypothermia or had hypothermia for $\leq 12$ hours. The decision to provide therapeutic hypothermia was based on the attending clinician's preference.

\section{MR acquisition and analysis}

MR scans were performed between 4 and 14 days of age on a 3T scanner (Philips, GE or Siemens) with harmonised protocols. We used MRS phantoms with known metabolite concentrations to cross-calibrate the scanners from each site prior to recruitment. All MR data were analysed centrally masked to the clinical details and outcome data (online supplementary eTable 1). The MR protocol and data analysis plan has been published previously. $^{7}$

\section{Neurodevelopmental assessment}

At 2 years of age, we performed a detailed neurological examination using Bayley-III. In cases where Bayley-III was not available, the British Association of Perinatal Medicine/Royal College of Paediatrics and Child Health working group classification was used instead. ${ }^{10}$

We considered any disability at 18-24 months of age as defined by the NICHD Neonatal Research Network as an adverse outcome. ${ }^{11}$ Mild disability was defined by a cognitive score of 70-84 alone, or a cognitive score $\geq 85$ and Gross Motor Function Classification System (GMFCS) level 1 or 2; a seizure disorder (without antiepileptic medication); or hearing deficit with ability to follow commands without amplification. Moderate disability was classified as: cognitive score from 70 to 84 and GMFCS level 2; active seizure disorder (receiving antiepileptic medication); or hearing deficit with the ability to follow commands after amplification. Finally, severe disability was defined as: cognitive score $<70$; GMFCS levels 3-5; blindness; or hearing impairment with inability to follow commands in spite of amplification.

\section{Statistical analysis}

Statistical analysis involved using a two-sided Student's t-test for parametric continuous variables and a Mann-Whitney U test for continuous non-parametric variables. Categorical variables were compared using a $\mathrm{X}^{2}$ test or Fisher's exact test. Analyses were performed with SPSS Statistics V.24 (IBM) and a p value of $<0.05$ was considered statistically significant.

\section{RESULTS}

A total of 47 babies with mild HIE were included in the study. Of these, $15(32 \%)$ were not cooled $(n=10)$ or were cooled for less than 12 hours $(n=5$, median duration of cooling 3 hours IQR $3-10)$, and 32 (68\%) had 72 hours of cooling. The baseline characteristics of cooled and non-cooled babies were similar apart from significantly lower $10 \mathrm{~min}$ Apgar scores in the cooled babies $(p=0.02$, table 1$)$. The hospital stay was significantly longer for the cooled babies $(p=0.04$, table 1$)$. Eight $(25 \%)$ of the cooled babies and $1(6 \%)$ of the non-cooled babies had a moderately abnormal aEEG within 6 hours of age $(p=0.2)$.

Four $(8.5 \%)$ babies with mild HIE and normal aEEG developed seizures after 6 hours of age (online supplementary eTable 2 ). Of these, one baby (case 4) was subsequently cooled for 72 hours, starting from 10 hours of age.

On conventional MRI, 29 out of the 47 (61\%) infants had abnormal white matter. The white matter lesion scores in the non-cooled babies (mean 1.3, SD 0.7) were significantly higher than in the fully cooled babies (mean 0.5 , SD $0.6, p=0.01$ ) (table 2). Abnormal white matter was seen in 50\% (16/32) of the cooled babies and $87 \%(13 / 15)$ of the non-cooled babies $(\mathrm{p}=0.02)$ (online supplementary eFigure 1$)$.

Only three infants had injury to the deep brain nuclei, two of whom had been cooled for 72 hours, while one was not cooled. There was no difference in injury scores between the two groups in the deep nuclei or cortex.

Six babies (one non-cooled and five cooled) were excluded from the MRS analysis due to poor quality data, or loss of data during transfer from the scanner. From the remaining MRS data, the thalamic ratios of NAA/Cr and NAA/Cho (adjusted for gestational age) were significantly higher in cooled babies than non-cooled babies (figure 1). After the exclusion of those infants who progressed to moderate HIE, the differences in brain injury between cooled and non-cooled babies persisted, with thalamic ratios of $\mathrm{NAA} / \mathrm{Cr}$ and $\mathrm{NAA} / \mathrm{Cho}$ significantly higher in fully cooled babies and white matter injury scores significantly higher in the non-cooled group.

No significant differences were found in the thalamic ratios of Lac/NAA between the two groups $(p=0.21)$ (figure 1$)$. Only two babies with mild HIE, who did not undergo cooling, had an elevated $(\geq 0.22)$ Lac/NAA ratio indicating a significant injury to the deep brain nuclei, and a high risk of adverse neurodevelopmental outcome. ${ }^{12}$ The Lac/NAA ratios were all below this threshold in the cooled babies.

A total of $40(85 \%)$ infants had neurodevelopmental outcome assessments for analysis (table 3 and figure 2). There was no significant difference in terms of baseline characteristics between the children who were lost at the follow-up and the remaining ones. Two non-cooled infants had mild disability at 2 years. One developed seizures at 33 hours of age, and was later diagnosed with hyperekplexia, confirmed by a homozygous novel missense 


\begin{tabular}{|c|c|c|}
\hline & $\begin{array}{l}\text { No cooling } \\
(n=15)\end{array}$ & $\begin{array}{l}72 \text { hours of } \\
\text { cooling }(n=32)\end{array}$ \\
\hline \multicolumn{3}{|l|}{ Antenatal history } \\
\hline Maternal diabetes & $2(13)$ & 0 \\
\hline Maternal hypertension & $1(6)$ & 3 \\
\hline Thyroid disease & 0 & 0 \\
\hline Reduced fetal movements & $2(13)$ & $2(6)$ \\
\hline CTG bradycardia & $2(13)$ & $10(31)$ \\
\hline Late decelerations & $1(6)$ & $2(6)$ \\
\hline Variable decelerations & $1(6)$ & $1(3)$ \\
\hline Prolonged rupture of membranes* & $2(13)$ & $6(18)$ \\
\hline \multicolumn{3}{|l|}{ Birth and resuscitation } \\
\hline Gestational age at birth (weeks) & $39.0(2.0)$ & $40(1.2)$ \\
\hline Birth weight $(\mathrm{kg})$ & $3.2(0.4)$ & $3.6(0.4)$ \\
\hline Cord pH & $7.1[0.5]$ & $6.9[0.2]$ \\
\hline Cord BE & $-9.2[6.8]$ & $-14.0[6.7]$ \\
\hline Temperature admission & $36.0(0.6)$ & $36.0(0.8)$ \\
\hline Apgar score (1 min) & $4[4]$ & $2[3]$ \\
\hline Apgar score (5 min) & $6[2]$ & $5[2]$ \\
\hline Apgar score (10 min) & $8[2] \dagger$ & $6[3] \dagger$ \\
\hline \multicolumn{3}{|l|}{ Neonatal course } \\
\hline Abnormal aEEG & $1(6)$ & $8(25)$ \\
\hline Invasive ventilation & $7(46)$ & $22(68)$ \\
\hline Non-invasive ventilation & $1(6)$ & $3(9)$ \\
\hline Hypotension requiring inotropes & $2(13)$ & $5(15)$ \\
\hline Abnormal clotting & $1(6)$ & $6(19)$ \\
\hline Decreased urine output & 0 & $3(9)$ \\
\hline $\begin{array}{l}\text { Maximum creatinine levels during the first } \\
72 \text { hours of age }\end{array}$ & $80(22)$ & $78(17)$ \\
\hline $\begin{array}{l}\text { Maximum CRP levels during the first } 72 \text { hours } \\
\text { of age }\end{array}$ & $22(44)$ & $23(23)$ \\
\hline Blood culture positivity & 0 & $1(3)$ \\
\hline Seizures after 6 hours of age & $4(27)$ & 0 \\
\hline Postnatal age at MRI (days) & $6.5[3.0]$ & $7.0[4.0]$ \\
\hline Hospital stay (days) & $5.5[6.2] \dagger$ & $8.0[2.0] \dagger$ \\
\hline
\end{tabular}

Data are mean (SD), $\mathrm{n}(\%)$, median [IQR].

*Suspected or confirmed rupture of membranes for more than 24 hours. $\dagger P<0.5$ (Mann-Whitney U test).

aEEG, amplitude integrated electroencephalography; CRP, C-reactive protein; CTG, cardiotocography; $\mathrm{BE}$, Base Excess; MRI, magnetic resonance imaging.

mutation SLC6A5 (case 3; online supplementary eTable 2). At the time of neurological assessment (18 months of age) there was mild motor disability without cerebral palsy and severe language delay. In the second case, the Bayley-III assessment (at 21 months of age) assigned a cognitive composite score of 80 (equivalent developmental age of 16 months), a language composite score of 74 (receptive communication equivalent developmental age of 16 months; expressive communication equivalent developmental age of 15 months) and a motor composite score of 82 (fine motor equivalent developmental age of 17 months; gross motor equivalent developmental age of 16 months). The infant had right arm monoplegia (GMFCS $=1)$.

\section{DISCUSSION}

In this multicentre prospective observational study on 47 babies with mild HIE at 6 hours of age, cooled babies had less white matter injury and improved thalamic metabolic indicators in comparison to non-cooled babies, despite having lower $10 \mathrm{~min}$
Table 2 MRI and MR spectroscopy in cooled and non-cooled groups with mild encephalopathy

\begin{tabular}{|c|c|c|}
\hline Conventional MRI & $\begin{array}{l}\text { No cooling } \\
(n=15)\end{array}$ & $\begin{array}{l}72 \text { hours of cooling } \\
(n=32)\end{array}$ \\
\hline \multicolumn{3}{|c|}{ Basal ganglia and thalami, n (\%) } \\
\hline Normal & $14(93.3)$ & $30(93.8)$ \\
\hline 1 & $1(6.7)$ & $2(6.3)$ \\
\hline 2 & 0 & 0 \\
\hline 3 & 0 & 0 \\
\hline \multicolumn{3}{|c|}{ Posterior limb of internal capsule, n (\%) } \\
\hline Normal & $15(100)$ & $32(100)$ \\
\hline 1 & 0 & 0 \\
\hline 2 & 0 & 0 \\
\hline 3 & 0 & 0 \\
\hline \multicolumn{3}{|l|}{ White matter, n (\%) } \\
\hline Normal & $2(13.3)$ & $16(50)$ \\
\hline 1 & $9(60)$ & $13(40.6)$ \\
\hline 2 & $4(26.7)$ & $3(9.4)$ \\
\hline 3 & 0 & 0 \\
\hline \multicolumn{3}{|l|}{ Cortex, n (\%) } \\
\hline Normal & $12(80)$ & $29(90.6)$ \\
\hline 1 & $3(20)$ & $3(9.4)$ \\
\hline 2 & 0 & 0 \\
\hline 3 & 0 & 0 \\
\hline MR spectroscopy & $\begin{array}{l}\text { No cooling } \\
(n=14)\end{array}$ & $\begin{array}{l}72 \text { hours of cooling } \\
(n=26)\end{array}$ \\
\hline Lac/NAA & $0.16(0.06)$ & $0.12(0.03)$ \\
\hline NAA/Cho & $0.67(0.08)^{*}$ & $0.89(0.11)^{*}$ \\
\hline $\mathrm{NAA} / \mathrm{Cr}$ & $1.42(0.11)^{*}$ & $1.64(0.18)^{*}$ \\
\hline
\end{tabular}

Data are number of patients or mean (SD).

${ }^{*} \mathrm{P}<0.001$ (Student's t-test).

Cho, total choline; $\mathrm{Cr}$, total creatinine; Lac, threonine+lactate; NAA,

$\mathrm{N}$-acetylaspartate+ $\mathrm{N}$-acetylaspartylglutamate.

Apgar scores. Four of the 15 non-cooled babies developed seizures and progressed to moderate HIE after 6 hours of age. The differences in brain injury between cooled and non-cooled babies persisted even after excluding these babies. The mean cognitive, motor and language scores at 2 years were similar in the cooled and non-cooled babies. However, 2 out of the 14 $(14.3 \%)$ cases in the non-cooling group had mild disability whereas none of the babies who were cooled had an adverse outcome at 2 years.

There has been only limited research so far describing the nature of injury in mild HIE and the effect of cooling therapy. Previous studies demonstrated that infants with mild HIE have predominately white matter injury whereas infants with moderate or severe HIE are more likely to have injury to the basal ganglia and thalami. ${ }^{13} 14$ This is likely to reflect different underlying disease mechanisms, since white matter injury is typically associated with subacute hypoxia, whereas injury to the deep nuclei is associated with acute profound asphyxia. ${ }^{15}$ However, in those studies none of the infants were cooled and therefore it was not possible to investigate the effect of cooling in mild HIE.

A previous study showed similar results with a lower incidence of brain injury on MRI in infants who were treated with wholebody cooling (4/13 (31\%)) when compared with those who did not receive cooling $(20 / 50(40 \%)) .{ }^{16}$ In that study, the authors found that the majority of neonates with mild HIE had an abnormal signal in the cortex regardless of whether or not they 

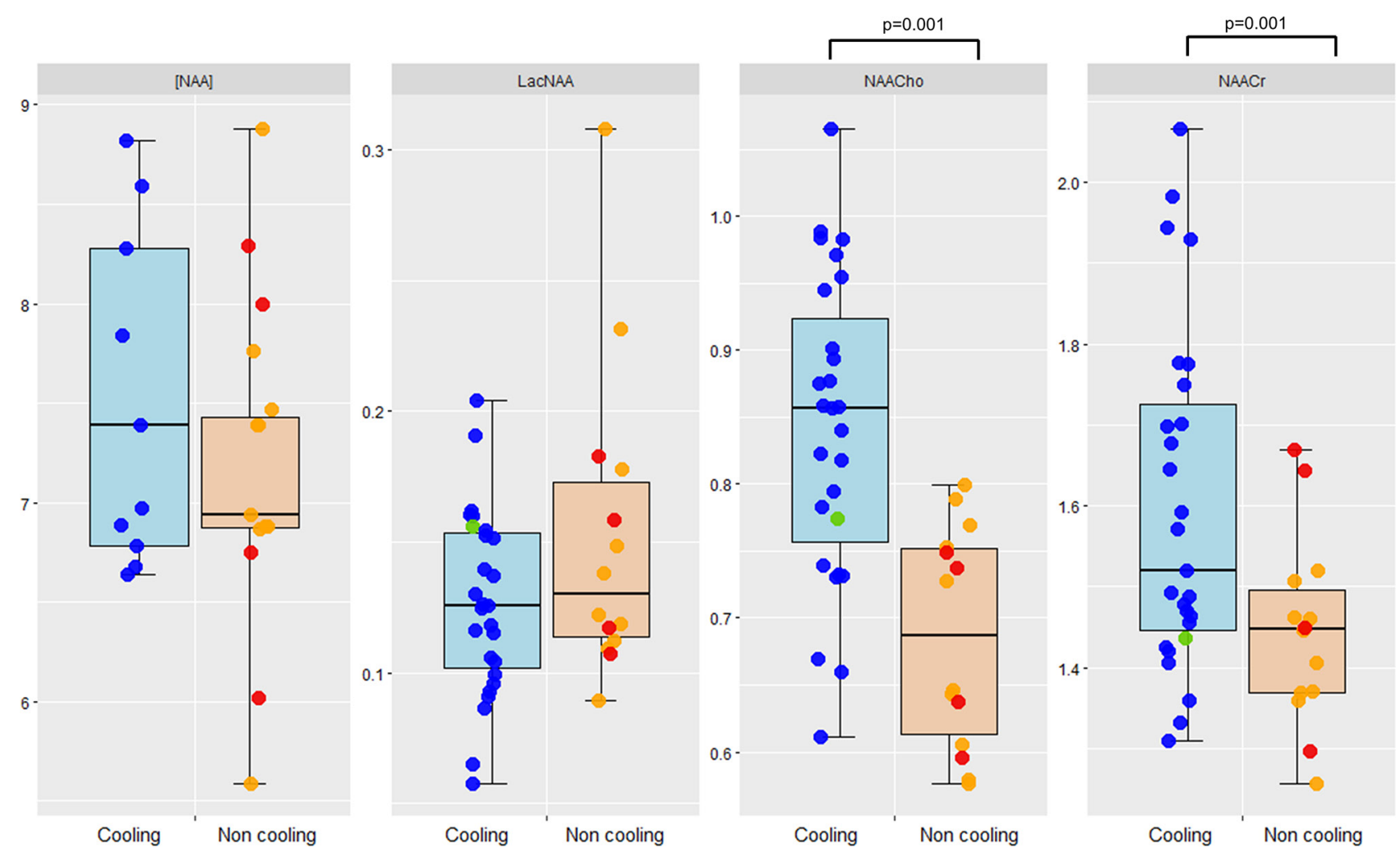

Figure 1 Dot plots (media, IQR) for MR spectroscopy measures in the cooled and non-cooled babies with mild encephalopathy. Blue dots indicate babies cooled for 72 hours, red dots indicate babies cooled for less than 24 hours, green dots indicate babies cooled after 6 hours, and orange dots indicate non-cooled babies. Lac/NAA, threonine+lactate/NA; NAA/Cho, N-acetylaspartate+N-acetylaspartylglutamate/total choline; NAA/Cr, $\mathrm{N}$-acetylaspartate+ $\mathrm{N}$-acetylaspartylglutamate/total creatinine.

were cooled. Of note, recent animal evidence suggests that therapeutic hypothermia preserves white matter when the preclinical model is subjected to mild hypoxia-ischaemia. ${ }^{17}$

In another retrospective study on 89 encephalopathic babies who received cooling therapy, Walsh et $a l^{18}$ reported a similar incidence of watershed injury in infants who had mild HIE $(18 / 48(36 \%))$ when compared with those with moderate $(11 / 32$ $(32 \%))$ or severe HIE (3/6 (50\%)). Only 2 (4\%) babies with mild HIE had abnormal signal in thalamic or basal ganglia in that study. Our data confirm these observations. We did not find any significant thalamic injury on the conventional MRI of babies with mild HIE, although some degree of white matter injury was seen in $50 \%(16 / 32)$ of the cooled babies and $87 \%$ $(13 / 15)$ of the non-cooled babies. In contrast, we found evidence of improved thalamic MRS markers (higher NAA/Cr and NAA/

Table 3 Neurodevelopmental outcomes at 2 years after mild encephalopathy

\begin{tabular}{lcc}
\hline & $\begin{array}{l}\text { No cooling } \\
(\mathbf{n}=\mathbf{1 4 )}\end{array}$ & $\begin{array}{l}\mathbf{7 2} \text { hours of cooling } \\
(\mathbf{n}=\mathbf{2 6})\end{array}$ \\
\hline Cognitive composite score & $106(13)$ & $105(15)$ \\
\hline Language composite score & $90(12)$ & $99(25)$ \\
\hline Motor composite score & $100(9)$ & $100(10)$ \\
\hline Motor composite score $<85$ & $1(7)$ & $1(3)$ \\
\hline Language composite score $<85$ & 4 & $2(8)$ \\
Cognitive composite score $<85$ & $1(7)$ & $1(3)$ \\
Cerebral palsy & $1(7)$ & 0 \\
\hline
\end{tabular}

Data are number (\%), mean (SD).

There was no statistically significant difference between the cooled and non-cooled groups.
Cho) in cooled babies with mild HIE, although Lac/NAA was normal in all babies except two. However, in spite of these differences in MRS, the neurodevelopmental outcome at 18-24 months was not different between the cooled and non-cooled infants. Although neurodevelopmental outcome is currently the gold standard of measuring efficacy in cooling therapy, our study was not powered to examine/detect any differences in the clinical outcomes.

Preclinical studies have observed neuroprotection with 24-48 hours of cooling, while very short periods or very long periods of cooling are not neuroprotective. ${ }^{19}$ Although the numbers were small, our study suggests that cooling for less than 24 hours is unlikely to be beneficial in mild HIE, as there was no difference in the metabolite profile of non-cooled and partially cooled babies. We previously demonstrated that there was MRI evidence of residual brain injury in $50 \%$ of the babies with mild HIE who were cooled for less than 24 hours, and $20 \%$ of them subsequently had adverse outcomes. ${ }^{20}$ Whether cooling for 24 or 48 hours is as good or better than cooling for 72 hours in mild HIE is not known.

The development of seizures and progression of mild HIE to moderate or severe HIE after 6 hours of age, and thus missing the therapeutic window for cooling, is a major concern for clinicians. Only 4 (8.5\%) babies with mild HIE progressed to moderate HIE after 6 hours in our study. Of these, only one had an adverse neurodevelopmental outcome at 2 years and was subsequently diagnosed with hyperekplexia. Gagne-Loranger et $a l^{16}$ reported progression of mild HIE to moderate in $6 \%$ of babies $(12 \%$ in the non-cooled group and $3 \%$ in the cooled group), which is consistent with the data from the Prospective Research in Mild Encephalopathy study, ${ }^{21}$ where a standardised 

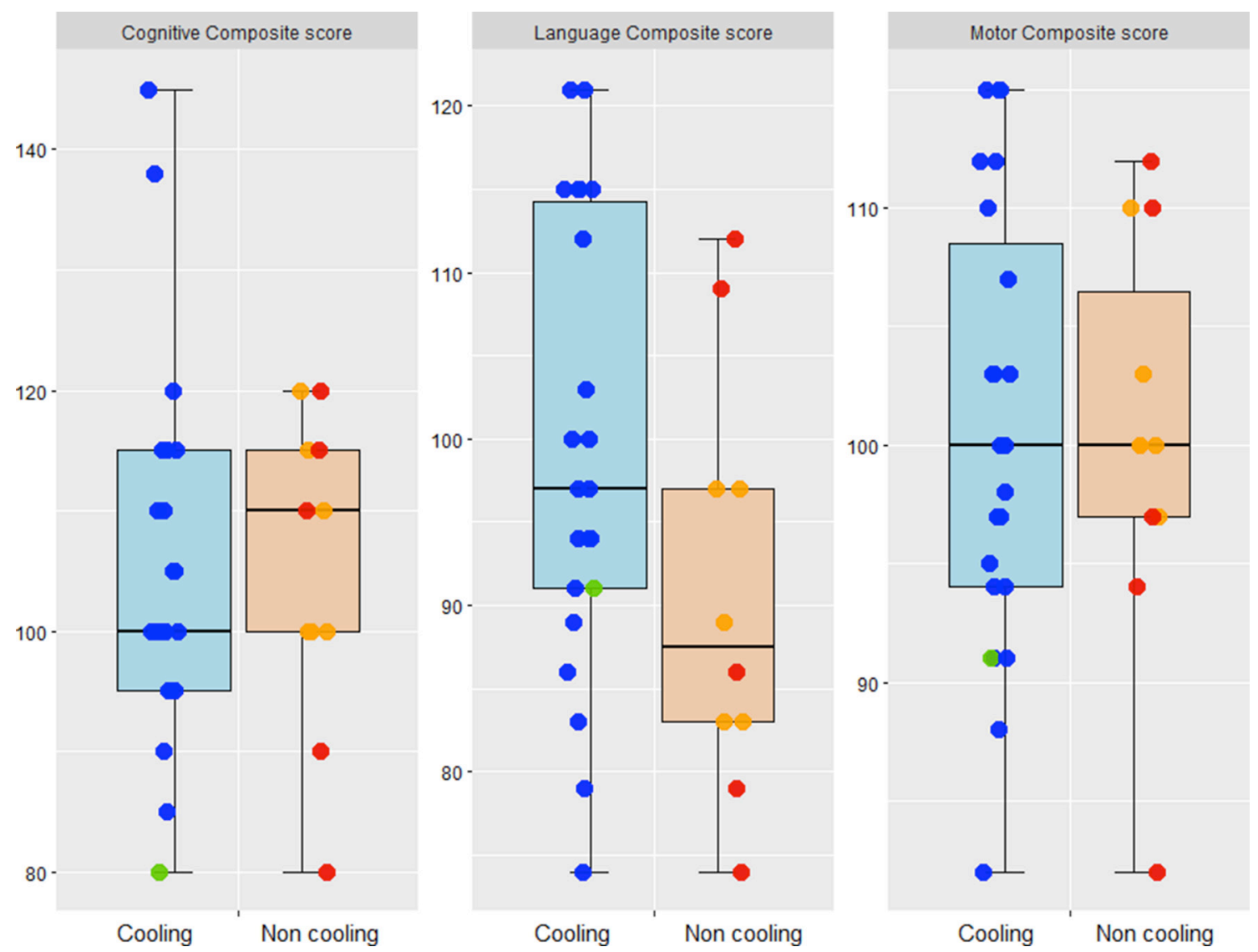

Figure 2 Dot plots (median, IQR) of the neurodevelopmental outcome scores in the cooled and non-cooled babies with mild encephalopathy. Blue dots indicate babies cooled for 72 hours, red dots indicate babies cooled for less than 24 hours, green dots indicate babies cooled after 6 hours, and orange dots indicate non-cooled babies.

neurological examination was performed. On the other hand, the Infant Cooling Evaluation (ICE) trial reported progression to moderate NE in 50\% of babies with mild HIE. The neurological examination was not standardised in the ICE trial, and it is possible that babies were misclassified as mild HIE at the initial examination. $^{22}$

Currently, the use of therapeutic hypothermia in babies with mild HIE is creeping into clinical practice despite a lack of evidence. In a recent survey, including 54 of the 68 UK cooling centres, $61 \%$ of the centres provided cooling for babies with mild HIE for the full 72 hours irrespective of any clinical improvement. ${ }^{3}$ Separately, recent surveys have reported a similar therapeutic drift, with almost half of the cooled babies in Australia and $15 \%$ of the cooled babies from the Children's Hospitals Neonatal Consortium in USA, lacking the features of moderate or severe HIE at initiation of cooling therapy. ${ }^{23} 24$

Although our study suggests a possible therapeutic benefit of cooling in mild HIE, caution is needed before drawing definitive conclusions, and a clinical decision to cool should not be taken lightly. Cooling therapy requires intensive care, often transfer to specialist centres and separation from parents, in addition to increased healthcare costs. One newborn piglet study has suggested cooling of the healthy brain may induce apoptosis, ${ }^{25}$ although this has not been replicated in other preclinical models. ${ }^{26}$ Given this lack of evidence and widespread variation in practice, there is an urgent need to investigate the impact of whole-body cooling in mild HIE infants in adequately powered randomised controlled trials.
This study has strengths and limitations. This is a small observational study, and hence no definitive conclusions on the therapeutic benefits of cooling can be made. Despite these, significant differences were identified in the metabolite peak area ratios of the cooled and non-cooled babies, suggesting that further evaluation of hypothermic neuroprotection in mild HIE is warranted. Our study was not powered to examine the clinical outcomes. Given the low event rates of adverse outcomes after mild HIE, thousands of babies would be required to detect improvements in neurodevelopmental outcomes. Study strengths include a robust prospective multicentre design, examinations performed by certified examiners, MRI and MRS analysis by a blinded central reader and scanner harmonisation prior to the start of the study.

\section{CONCLUSION}

Our study demonstrates that babies with mild HIE have less white matter and metabolic brain injury after therapeutic hypothermia, suggesting that cooling may have a neuroprotective effect in these infants.

Contributors PM acquired and analysed the data, performed neurodevelopmental outcome assessments and wrote the first draft of the manuscript. PJL acquired and analysed the MR spectroscopy data and wrote the first draft of the manuscript. $N L$, JT, RP, RS, VO, JM, GA, UK, VS and AS supported the data acquisition and interpretation. SS assisted in designing the study and interpretation of the data. ST conceived the idea, designed the study, interpreted the data and supervised all aspects of the work. All authors were involved in editing of the manuscript for important intellectual content and approved the final version submitted for publication. 
Funding The study was funded by the National Institute for Health Research and was supported by the NIHR Biomedical Research Centre based at Imperial College London, UK. PM is funded by a Medical Research Council doctoral fellowship. PJL is funded by a National Institute for Health Research clinical trials fellowship. VO is funded by the National Institute for Health Research doctoral fellowship.

Disclaimer The views expressed are those of the author(s) and not necessarily those of the NIHR or the Department of Health.

Competing interests None declared.

Patient consent Not required.

Ethics approval The North London Research Ethics Committee (11/H0717/6).

Provenance and peer review Not commissioned; externally peer reviewed.

Data sharing statement This will be available from the corresponding author.

Open access This is an open access article distributed in accordance with the Creative Commons Attribution 4.0 Unported (CC BY 4.0) license, which permits others to copy, redistribute, remix, transform and build upon this work for any purpose, provided the original work is properly cited, a link to the licence is given, and indication of whether changes were made. See: https://creativecommons.org/ licenses/by/4.0/.

\section{REFERENCES}

1 Jacobs SE, Berg M, Hunt R, et al. Cooling for newborns with hypoxic ischaemic encephalopathy. Cochrane Database Syst Rev 2013:CD003311.

2 Conway JM, Walsh BH, Boylan GB, et al. Mild hypoxic ischaemic encephalopathy and long term neurodevelopmental outcome - A systematic review. Early Hum Dev 2018;120:80-7.

3 Oliveira V, Singhvi DP, Montaldo P, et al. Therapeutic hypothermia in mild neonatal encephalopathy: a national survey of practice in the UK. Arch Dis Child Fetal Neonatal Ed 2018;103:F388-90.

4 Robertson NJ, Thayyil S, Cady EB, et al. Magnetic resonance spectroscopy biomarkers in term perinatal asphyxial encephalopathy: from neuropathological correlates to future clinical applications. Curr Pediatr Rev 2014;10:37-47.

5 Walsh BH, Inder TE. MRI as a biomarker for mild neonatal encephalopathy. Early Hum Dev 2018;120:75-9

6 Williams CE, Gunn AJ, Mallard C, et al. Outcome after ischemia in the developing sheep brain: an electroencephalographic and histological study. Ann Neurol 1992;31:14-21.

7 Lally PJ, Pauliah S, Montaldo P, et al. Magnetic Resonance Biomarkers in Neonatal Encephalopathy (MARBLE): a prospective multicountry study. BMJ Open 2015;5:e008912.

8 Shankaran S, Laptook AR, Ehrenkranz RA, et al. Whole-body hypothermia for neonates with hypoxic-ischemic encephalopathy. N Engl J Med 2005;353:1574-84.

9 Prempunpong C, Chalak LF, Garfinkle J, et al. Prospective research on infants with mild encephalopathy: the PRIME study. J Perinatol 2018;38:80-5.
10 Morioka T, Kalehua AN, Streit WJ. Progressive expression of immunomolecules on microglial cells in rat dorsal hippocampus following transient forebrain ischemia. Acta Neuropathol 1992;83:149-57.

11 Shankaran S, Laptook AR, Pappas A, et al. Effect of Depth and Duration of Cooling on Death or Disability at Age 18 Months Among Neonates With Hypoxic-Ischemic Encephalopathy: A Randomized Clinical Trial. JAMA 2017;318:57-67.

12 Lally PJ, Montaldo P, Oliveira V, et al. Magnetic resonance spectroscopy assessment of brain injury after moderate hypothermia in neonatal encephalopathy: a prospective multi-centre study. Lancet Neurology. In Press. 2018.

13 Miller SP, Ramaswamy V, Michelson D, et al. Patterns of brain injury in term neonatal encephalopathy. J Pediatr 2005; 146:453-60.

$14 \mathrm{Am} \mathrm{L}$, Chau V, Poskitt KJ, et al. White matter injury in term newborns with neonatal encephalopathy. Pediatr Research 2009;65:85-9.

15 Sie LT, van der Knaap MS, Oosting J, et al. MR patterns of hypoxic-ischemic brain damage after prenatal, perinatal or postnatal asphyxia. Neuropediatrics 2000;31:128-36.

16 Gagne-Loranger M, Sheppard M, Ali N, et al. Newborns Referred for Therapeutic Hypothermia: Association between Initial Degree of Encephalopathy and Severity of Brain Injury (What About the Newborns with Mild Encephalopathy on Admission?). Am J Perinatol 2016;33:195-202.

17 Koo E, Sheldon RA, Lee BS, et al. Effects of therapeutic hypothermia on white matter injury from murine neonatal hypoxia-ischemia. Pediatr Res 2017;82:518-26.

18 Walsh BH, Neil J, Morey J, et al. The Frequency and Severity of Magnetic Resonance Imaging Abnormalities in Infants with Mild Neonatal Encephalopathy. J Pediatr 2017; 187:26-33.

19 Davidson JO, Draghi V, Whitham $S$, et al. How long is sufficient for optimal neuroprotection with cerebral cooling after ischemia in fetal sheep? J Cereb Blood Flow Metab 2018;38:1047-59.

20 Lally PJ, Montaldo P, Oliveira V, et al. Residual brain injury after early discontinuation of cooling therapy in mild neonatal encephalopathy. Arch Dis Child Fetal Neonatal Ed 2018;103:F383-7.

21 Chalak LF, Nguyen KA, Prempunpong C, et al. Prospective research in infants with mild encephalopathy identified in the first six hours of life: neurodevelopmental outcomes at 18-22 months. Pediatr Res 2018 (Epub ahead of print 13 Sep 2018).

22 Jacobs SE, Morley CJ, Inder TE, et al. Whole-body hypothermia for term and near-term newborns with hypoxic-ischemic encephalopathy: a randomized controlled trial. Arch Pediatr Adolesc Med 2011;165:692-700.

23 Mehta S, Joshi A, Bajuk B, et al. Eligibility criteria for therapeutic hypothermia: From trials to clinical practice. J Paediatr Child Health 2017;53:295-300.

24 Massaro AN, Murthy K, Zaniletti I, et al. Short-term outcomes after perinatal hypoxic ischemic encephalopathy: a report from the Children's Hospitals Neonatal Consortium HIE focus group. J Perinatol 2015;35:290-6.

25 Wang $B$, Armstrong JS, Reyes $M$, et al. White matter apoptosis is increased by delayed hypothermia and rewarming in a neonatal piglet model of hypoxic ischemic encephalopathy. Neuroscience 2016;316:296-310.

26 Auriat AM, Klahr AC, Silasi G, et al. Prolonged hypothermia in rat: a safety study using brain-selective and systemic treatments. Ther Hypothermia Temp Manag 2012;2:37-43. 